\title{
Relationship Between Renal Function and Renal Artery Involvement in Acute Debakey Type I Aortic Dissection
}

\author{
Yi-Peng Ge, MD, ${ }^{1}$ Cheng-Nan Li, MD,${ }^{1}$ Yu Li, MD,${ }^{2}$ Jun-Ming Zhu, MD, ${ }^{1}$ Yong-Min Liu, MD,${ }^{1}$ \\ Jun Zheng, MD, ${ }^{1}$ Li-Zhong Sun, $\mathrm{MD}^{1}$ \\ ${ }^{1}$ Department of Cardiovascular Surgery, Beijing Aortic Disease Center, Beijing Anzhen Hospital, Capital Medical University, Beijing, \\ China; ${ }^{2}$ Department of Radiology, Beijing Anzhen Hospital, Capital Medical University, Beijing, China
}

\section{ABSTRACT}

Background: The aim of this study was to analyse the differences in renal function among various types of renal artery involvement in acute Debakey Type I aortic dissection.

Methods: From January 2016 to January 2018, 304 consecutive patients with acute Debakey type I aortic dissection with renal artery involvement were included. According to computed tomography angiography (CTA) findings, renal artery involvement on one side can be classified into four types: type $\mathrm{A}$, in which a large intimal tear is near the renal artery orifice; type $\mathrm{B}$, the orifice of the renal artery originates entirely from the false lumen; type $\mathrm{C}$, the orifice of the renal artery originates entirely from the true lumen; and type D, a renal artery dissection is observed. All patients underwent aortic repair.

Results: The average age was $46.98 \pm 10.64$ years. The types of bilateral renal artery involvement were as follows: $\mathrm{AB}$ type, four patients $(1.32 \%)$; AC type, 38 patients $(12.50 \%)$; $\mathrm{AD}$ type, three patients (0.99\%); BB type, 13 patients $(4.28 \%)$; BC type, 140 patients (46.05\%); BD type, four patients $(1.32 \%)$; CC type, 76 patients $(25.00 \%)$; and CD type, 26 patients $(8.55 \%)$. One-way ANOVA showed that there was no significant difference in serum creatinine $(P=.57)$ and creatinine clearance rate $(P=.08)$ between the groups. A statistically significant difference in age, gender, body mass index, hypertension history and aortic dissection onset time also was not observed $(P>.05)$. The overall incidence of KDIGO acute kidney injury (AKI) was $49.67 \%$. There was no significant difference in AKI incidence between different types of renal artery involvement after aortic surgery $(P=.39)$. For patients needing renal replacement therapy, CTA showed that enhancement of renal cortex in the arterial phase was low and the boundary between the cortex and medulla was unclear in bilateral kidneys.

Conclusion: The types of renal artery involvement did not affect renal function in the acute phase.

Received April 17, 2020; accepted May 28, 2020.

Correspondence: Yi-Peng Ge, MD, Beijing Aortic Disease Center, Beijing Anzhen Hospital, Capital Medical University, Anzhen Road 2\#, Chaoyang District, Beijing, China 100029; 86-10-64456579; fax 86-10-64456588 (e-mail: yipengge@126.com).

\section{INTRODUCTION}

Debakey type I aortic dissection often involves the renal arteries. However, no study has reported the proportion of different types of renal artery involvement or explored whether different types of renal artery involvement can affect serum creatinine levels. The main purpose of this study was to establish the classification of renal artery involvement in Debakey type I aortic dissection patients by using aortic computed tomography angiography (CTA) as an imaging tool and to analyse the differences in renal function among various types of renal artery involvement.

\section{PATIENTS AND METHODS}

Patient data: Between January 2016 to January 2018, data from 304 consecutive patients diagnosed with Debakey type I aortic dissection retrospectively were collected from Beijing Anzhen Hospital. Patients who suffered chronic renal insufficiency were excluded. All the patients underwent aortic repair. The Ethics Committee of Beijing Anzhen Hospital

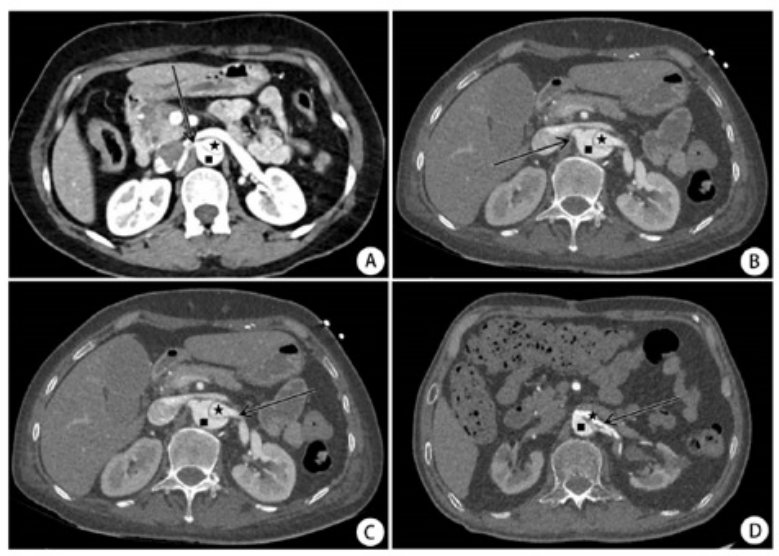

Figure 1. Classification of renal artery involvement. A, Right renal artery involvement, type A; B, Right renal artery involvement, type B; C, Left renal artery involvement, type C; $D$, Left renal artery involvement, type D. Arrow indicates the orifice of the renal artery. Square indicates the false lumen. Asterisk indicates the true lumen. 
Table 1. Renal artery involvement of right and left renal artery

\begin{tabular}{lcc}
\hline Type & Right renal artery $(\mathrm{N}=304)$ & Left renal artery $(\mathrm{N}=304)$ \\
\hline A & $34(11.18 \%)$ & $11(3.62 \%)$ \\
B & $65(21.38 \%)$ & $109(35.86 \%)$ \\
C & $195(64.14 \%)$ & $61(52.96 \%)$ \\
$D$ & $10(3.29 \%)$ & $23(7.57 \%)$ \\
\hline
\end{tabular}

approved this retrospective study and waived the need for consent from individual patients.

Classification of renal artery involvement: According to CTA results, renal artery involvement on one side can be classified into four types: type A, in which a large intimal tear is near the renal artery orifice, it is difficult to distinguish whether the renal artery originates from a true or false lumen (Figure 1A); type B, the orifice of the renal artery originates entirely from the false lumen (Figure 1B); type $\mathrm{C}$, the orifice of the renal artery originates entirely from the true lumen (Figure 1C); and type D, a renal artery dissection is observed, and renal artery intima can be observed (Figure 1D).

CT image acquisition: All studies were performed on a 128-slice dual-source computed tomography system (SOMATOM Definition Flash, Siemens Healthcare, Forchheim, Germany). Contrast-enhanced scans were performed from the thoracic inlet to the pubic symphysis. All CT imaging data were acquired while the patient held his or her breath in deep inspiration to eliminate respiratory motion artefacts. The scanning parameters were as follows: slice collimation of $128 \times 0.6 \mathrm{~mm}$ with a $\mathrm{z}$-flying focal spot, gantry rotation time of $280 \mathrm{~ms}$, pitch of 3.2, and tube voltage of $120 \mathrm{kV}$. Contrast medium (CM) was injected with an 18-gauge needle through the right antecubital vein by a dual-syringe power injector. Regions of interest (ROIs) were placed within the descending aorta to trigger the scan. The total volume of injected CM was adjusted, according to body mass index ( $1 \mathrm{~mL}$ of $\mathrm{CM}$ per $\mathrm{kg}$ body weight) followed by $30 \mathrm{~mL}$ of saline solution. The injection rate for $\mathrm{CM}$ and saline solution was $4-5 \mathrm{~mL} / \mathrm{s}$ for all subjects. Transverse images were reconstructed with a slice thickness of $1 \mathrm{~mm}$ in $1-\mathrm{mm}$ increments. The datasets were transferred to an external workstation (Syngo Multi-Modality Work Place, CT 2011A, Siemens Healthcare) for further image analysis. Using the axial data, one cardiac radiologist reconstructed and analysed the images with the volume rendering technique, maximum intensity projection, and multiplanar reconstruction.

Statistical analysis: All tests were two-sided and significant if the $P$-value was $<.05$. The variables were analyzed using one-way ANOVA between different types of renal artery involvement.

Repeated measures ANOVA was used to analyze the time trend changes in renal function between different groups. Data were analyzed using SPSS software, version 22.0 (SPSS Inc).

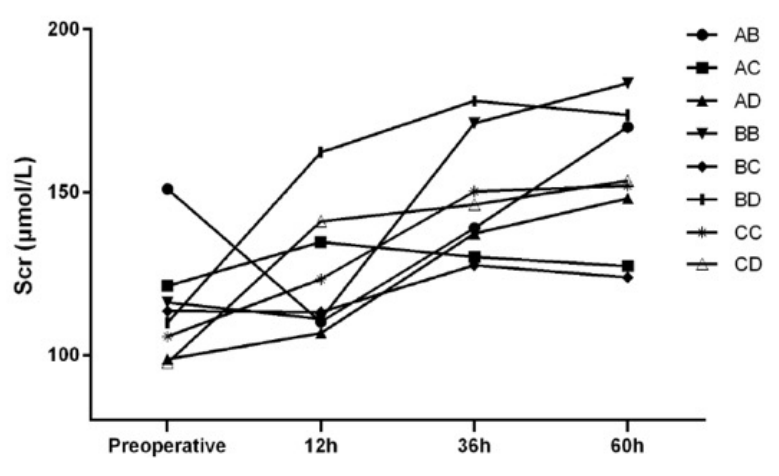

Figure 2. Time trends of sCr before and after aortic repair

\section{RESULTS}

The average age was $46.98 \pm 10.64$ years. A total of 243 patients $(79.93 \%)$ were male. The distribution of renal artery involvement was 45 arteries with type $\mathrm{A}(7.40 \%), 174$ arteries with type B (28.62\%), 356 arteries with type C (58.55\%), and 33 arteries with type $\mathrm{D}(5.43 \%)$. The distribution of types of left and right renal artery involvement is shown in Table 1. (Table 1) The types of bilateral renal artery involvement were as follows: $\mathrm{AB}$ type, four patients $(1.32 \%)$ with a serum creatinine $(\mathrm{sCr})$ level of $65.25 \pm 21.84 \mu \mathrm{mol} / \mathrm{L}$ and creatinine clearance rate (Ccr) level of $150.95 \pm 99.39 \mathrm{ml} / \mathrm{min}$; AC type, 38 patients $(1.50 \%)$ with a sCr level of $79.20 \pm 20.21 \mu \mathrm{mol} / \mathrm{L}$ and Ccr level of $121.25 \pm 33.69 \mathrm{ml} / \mathrm{min}$; AD type, three patients $(0.99 \%)$ with a sCr level of $77.70 \pm 23.49 \mu \mathrm{mol} / \mathrm{L}$ and Ccr level of $98.77 \pm 36.07 \mathrm{ml} / \mathrm{min}$; BC type, 140 patients (46.05\%) with a sCr level of $84.70 \pm 30.65 \mu \mathrm{mol} / \mathrm{L}$ and Ccr level of $113.53 \pm$ $37.74 \mathrm{ml} / \mathrm{min}$; BD type, four patients $(1.32 \%)$ with a sCr level of $87.78 \pm 21.86 \mu \mathrm{mol} / \mathrm{L}$ and Ccr level of $110.00 \pm 45.12 \mathrm{ml} /$ min; CC type, 76 patients (25.00\%) with a sCr level of 87.94 $\pm 33.45 \mu \mathrm{mol} / \mathrm{L}$ and Ccr level of $105.78 \pm 36.69 \mathrm{ml} / \mathrm{min}$; and CD type, 26 patients $(8.55 \%)$ with sCr level of $93.51 \pm 41.57$ $\mu \mathrm{mol} / \mathrm{L}$ and Ccr level of $97.72 \pm 32.38 \mathrm{ml} / \mathrm{min}$.

One-way ANOVA showed that there was no significant difference in $\mathrm{s} \operatorname{Cr}(P=.57)$ and $\operatorname{Ccr}(P=.08)$ between the groups. A statistically significant difference in age, gender, body mass index, hypertension history and aortic dissection onset time also was not observed $(P>.05)$ (Table 2). The overall incidence of KDIGO acute kidney injury (AKI) after aortic surgery was $49.67 \%$. There was no significant difference in AKI incidence between the groups $(P=.39)$. We collected $\mathrm{sCr}$ and $\mathrm{Ccr}$ at four time points (pre-operation, 12 hours after surgery, 36 hours after surgery, and 60 hours after surgery). Repeated Measures ANOVA also showed that there was no significant difference in $\mathrm{sCr}$ and $\mathrm{Ccr}$ between eight groups (betweensubject) $(P=.47)$. However, a statistically significant difference was observed in time trends of $\mathrm{sCr}$ and $\mathrm{Ccr}$ at four time points (within-subject) $(P>.05)$ (Figure 2).

A total of 151 patients (49.67\%) suffered AKI. Most AKI occurred within 12 hours after aortic repair (151 patients, 
Table 2. Main clinical characteristics stratified by bilateral renal artery involvement

\begin{tabular}{|c|c|c|c|c|c|c|c|c|c|}
\hline Age (years)* & $45.75 \pm 12.04$ & $45.74 \pm 10.43$ & $46.33 \pm 8.39$ & $45.00 \pm 13.08$ & $46.14 \pm 10.58$ & $40.50 \pm 11.90$ & $49.08 \pm 10.51$ & $49.42 \pm 10.04$ & .36 \\
\hline Hypertension & $2(50.00 \%)$ & $29(76.32 \%)$ & $1(33.33 \%)$ & $8(61.54 \%)$ & $99(70.71 \%)$ & $3(75.00 \%)$ & $64(84.21 \%)$ & $22(84.62 \%)$ & .08 \\
\hline DM & $0(0.00 \%)$ & $2(5.26 \%)$ & $1(33.33 \%)$ & $0(0.00 \%)$ & $6(4.29 \%)$ & $0(0.00 \%)$ & $3(3.95 \%)$ & $2(7.69 \%)$ & .46 \\
\hline $\operatorname{LVEF}(\%)^{*}$ & $62.25 \pm 5.80$ & $60.58 \pm 6.40$ & $63.33 \pm 2.89$ & $62.75 \pm 6.72$ & $61.14 \pm 4.78$ & $57.50 \pm 2.08$ & $62.22 \pm 5.50$ & $61.58 \pm 4.74$ & .31 \\
\hline Onset time $(h) \dagger$ & $\begin{array}{c}36 \\
(18.75,66)\end{array}$ & $\begin{array}{c}24 \\
(15.75,48.00)\end{array}$ & 24 & $\begin{array}{c}20.00 \\
(14.00,36.00)\end{array}$ & $\begin{array}{c}24.00 \\
(12.00,48.00)\end{array}$ & $\begin{array}{c}12.50 \\
(5.50,22.50)\end{array}$ & $\begin{array}{c}24.00 \\
(18.00,66.00)\end{array}$ & $24(20,48)$ & .30 \\
\hline CPB time* & $197.75 \pm 38.27$ & $215.21 \pm 54.88$ & $197.67 \pm 25.32$ & $199.50 \pm 30.56$ & $200.72 \pm 41.84$ & $221.00 \pm 39.97$ & $213.17 \pm 49.02$ & $203.96 \pm 48.18$ & .51 \\
\hline $\operatorname{Scr}(\mu \mathrm{mol} / \mathrm{L})^{*}$ & $65.25 \pm 21.84$ & $79.20 \pm 20.21$ & $77.70 \pm 23.49$ & $84.97 \pm 26.94$ & $84.70 \pm 30.65$ & $87.78 \pm 21.86$ & $87.94 \pm 33.45$ & $93.51 \pm 41.60$ & .57 \\
\hline
\end{tabular}

Values in parentheses are percentages. *Values are the mean \pm SD. †Value is Median (P25,P75). DM, diabetes mellitus; BMI, body mass index; LVEF, left ventricular ejection fraction; CPB, cardiopulmonary bypass; $\mathrm{s} C r$, serum creatinine; $\mathrm{Ccr}$, creatinine clearance rate

$70.20 \%)$. Forty patients (26.49\%) occurred between 12-36 hours, three patients (1.99\%) between 36-60 hours, and two patients $(1.32 \%) 60$ hours later. There was no significant difference between eight of the groups $(P=.49)$ (Table 3).

A total of 35 patients needed renal replacement therapy (RRT) after aortic repair. Of these 35 patients, seven patients died in the hospital. Nineteen patients stopped RRT within two weeks after surgery. Other patients stopped RRT within three months after surgery. We reexamined CTA in nine patients, who needed RRT before being discharged from the hospital. For these nine patients, the left and right renal artery were shown clearly (Figure 3B). However, the enhancement of renal cortex in the arterial phase was low, and the boundary between the cortex and medulla was unclear in bilateral kidneys. This phenomenon was not related to the types of classification of renal artery involvement. For other patients who did not need RRT, we routinely reexamined aortic CTA and did not find a similar phenomenon, including KDIGO stage 3 patients without RRT. Three months later after surgery, of these nine patients who needed RRT and also received CTA scan before being discharged from the hospital, five patients had completely normal CTA scan and CTA performance (Figure 3C).

\section{DISCUSSION}

Acute Debakey type I aortic dissection is a catastrophic disease that often requires emergency surgery to save lives. In the past 10 years, with the advancement of surgical technology, the surgery mortality rate greatly has been reduced. However, the incidence of complications, especially AKI, has remained high, ranging from $18 \%$ to $77 \%$ [Ko 2015; Zhou 2018; Englberger 2011]. Renal malperfusion before the operation also has been reported as a strong risk factor for mortality [Ma 2014; Zindovic 2019; Hoogmoed 2019]. These patients have high medical expenses, stay in the hospital for a long time, and have a poor prognosis.

Unlike the celiac artery and superior mesenteric artery, the renal artery has two main branches: the left and right renal arteries, and an accessory renal artery is not uncommon. This characteristic undoubtedly increases the difficulty of preoperative evaluation of renal malperfusion. At present, aortic CTA is mainly used to evaluate the range and extent of renal artery involvement. The advantages of aortic CTA are objective and can provide good image quality. However, the disadvantages of aortic CTA are that it can reflect only static renal artery involvement and cannot reflect the dynamic perfusion of the renal artery. In theory, the dynamic motion of the intimal flap may have a significant impact on renal perfusion. Based on the above considerations, our team tried to determine the renal resistance index (RRI) by renal artery ultrasound, with the goal of evaluating renal malperfusion by RRI [Wu 2017].

However, renal ultrasonography had the following limitations: In addition to the influence of mean arterial pressure and $\mathrm{PaCO} 2$ on the RRI, breathing and body position also markedly influence the results [Wu 2017; Bossard 2011]. The duration to complete renal artery ultrasound examination is long, which might increase the risk of rupture of the aortic dissection. Based on the above shortcomings of renal ultrasonography, we still prefer aortic CTA as the main imaging tool for the evaluation of renal malperfusion.

Chan et al reported that renal artery malperfusion is classified into three types [Chan 2012]. He concluded that type 3 perfusion, which was defined as a renal artery perfused exclusively from the false lumen, is most frequently detected in the left renal artery and that such perfusion was significantly related to the development of renal atrophy [Chan 2012]. The limitation of this classification is that it did not include 
Table 3. AKI occurrence in different time after aortic repair for different renal artery involvement types

\begin{tabular}{lcccc}
\hline Types & $\begin{array}{c}0-12 \mathrm{~h} \\
(\mathrm{~N}=106)\end{array}$ & $\begin{array}{c}12-36 \mathrm{~h} \\
(\mathrm{~N}=40)\end{array}$ & $\begin{array}{c}36-60 \mathrm{~h} \\
(\mathrm{~N}=3)\end{array}$ & $\begin{array}{c}\text { 60h later } \\
(\mathrm{N}=2)\end{array}$ \\
\hline $\mathrm{AB}$ & 3 & 0 & 0 & 0 \\
$\mathrm{AC}$ & 15 & 5 & 0 & 0 \\
$\mathrm{AD}$ & 1 & 0 & 0 & 0 \\
$\mathrm{BB}$ & 5 & 4 & 0 & 0 \\
$\mathrm{BC}$ & 44 & 13 & 2 & 1 \\
$\mathrm{BD}$ & 2 & 0 & 0 & 0 \\
$\mathrm{CC}$ & 25 & 16 & 0 & 0 \\
$\mathrm{CD}$ & 11 & 2 & 1 & 1 \\
\hline
\end{tabular}

patients with large intimal tears near the orifice of the renal artery, which was defined as type A perfusion by our team. The proportion of type A perfusion was as high as $8.28 \%$, and thus, it is reasonable to separately classify this type.

To our knowledge, this is first report that described aortic CTA characteristics of patients needing RRT after aortic surgery. It is interesting that for these patients needing RRT, the left and right renal arteries were clearly shown, but enhancement of the renal cortex in the arterial phase was low and the boundary between the cortex and medulla was unclear in the bilateral kidneys. This phenomenon was not related to the types of classification of renal artery involvement. This might suggest that classification of renal artery involvement might not be a potential risk factor that leads to AKI. At present, the mechanism of AKI is not clear. According to the imaging characteristics of CTA, renal microcirculatory dysfunction may be the reason for AKI. It is believed that CPB can lead to the formation of circulating microaggregates of adhesions among leukocytes, activated platelets, and endothelial cells and is an important risk factor of AKI [Kumar 2012; Rosner 2006; Kumar 2011; Park 2017; Bruins 1997; Garg 2014]. Most recently, more researchers have found that blood transfusion could be another risk factor of AKI [Karkouti 2012; Kilic 2014; Kindzelski 2018]. Blood transfusion leads to increased levels of free hemoglobin, cytokines, and iron that further damage the glomerular filtration apparatus [Kindzelski 2018; Ho 2009; Vercaemst 2008; Khan 2014]. Renal microcirculatory dysfunction might be the pathway of damage of $\mathrm{CPB}$ and other risk factors. This hypothesis should be tested in future studies.

Currently, the use of sCr and urine volume to assess renal function is controversial [Wasung 2015; Haase 2015; Redahan 2017]. Elevated sCr levels often are observed at 48-72 hours after renal injury, with low sensitivity and specificity. After the glomerular filtration rate has decreased by more than $50 \%$, the sCr level subsequently increases by a significant amount. Therefore, the level of sCr cannot accurately reflect renal malperfusion at an early stage. In addition, the increase in sCr level is influenced by other factors,

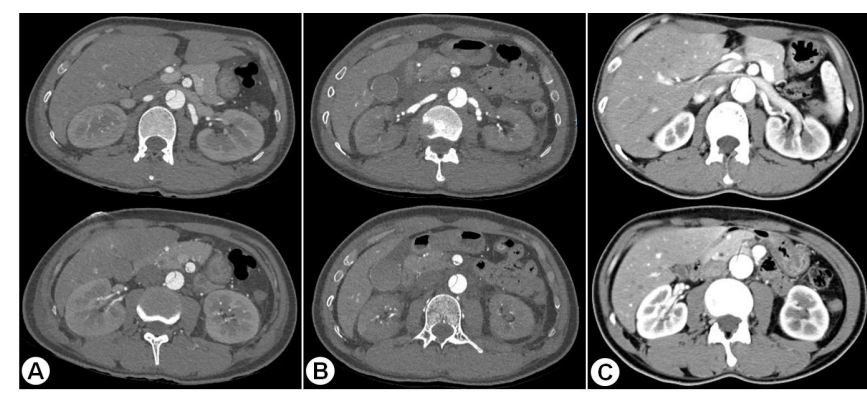

Figure 3. CTA preoperative, postoperative and follow up. A, Preoperative CTA. Renal function was normal. Enhancement of renal cortex was high. The boundary between cortex and medulla was clear. B, Postoperative CTA. The patient suffered from AKI and needed RRT. The left and right renal artery were shown clearly, but enhancement of renal cortex in arterial phase was low and the boundary between cortex and medulla was unclear in bilateral kidneys. C, Follow-up CTA. Three months later. Renal function recovered. Enhancement of renal cortex was high. The boundary between cortex and medulla was clear.

such as age, nutritional status, and chronic renal failure. Therefore, it is reasonable that there is no significant difference in sCr level among the four types of renal malperfusion observed in this study. Interestingly, Wang et al. followed the patients with renal malperfusion caused by aortic dissection and found that the degree of renal atrophy was not correlated with the change in the glomerular filtration rate; in addition, $\gamma 2$ was only 0.04 [Wang 2018]. This finding indicates that the existing laboratory indicators of renal function have significant limitations in evaluating malperfusion caused by aortic dissection. Although many studies have explored neutrophil gelatinase-associated lipocalin (NGAL), cystatin C, and kidney injury molecule-1 (KIM-1) as new biomarkers for renal injury [Klein 2018], these new acute kidney injury biomarkers have not yet been evaluated in the field of aortic dissection.

\section{LIMITATIONS}

First, the sample size is small, and the research lacks data of long-term follow up. Second, only a few patients needing RRT received a CTA scan. Finally, we lack basic research, which can explain that interesting imaging phenomenon.

\section{CONCLUSIONS}

The three most common types of bilateral renal artery involvement were $\mathrm{BC}$ type, $\mathrm{CC}$ type, and $\mathrm{AC}$ type. The types of renal artery involvement did not affect renal function in the acute phase.

\section{ACKNOWLEDGMENT}

This study was supported by Beijing Hospital Authority Youth Program (No. QML20190608). 


\section{REFERENCES}

Bossard G, Bourgoin P, Corbeau JJ, Huntzinger J, Beydon L. 2011. Early detection of postoperative acute kidney injury by Doppler renal resistive index in cardiac surgery with cardiopulmonary bypass. Br J Anaesth 107(6):891-8.

Bruins P, te Velthuis H, Yazdanbakhsh AP, Jansen PG, van Hardevelt FW, de Beaumont EM, et al. 1997. Activation of the complement system during and after cardiopulmonary bypass surgery: postsurgery activation involves C-reactive protein and is associated with postoperative arrhythmia. Circulation 96(10):3542-8.

Chan WH, Huang YC, Weng HH, Ko SF, Chu JJ, Lin PJ, et al. 2012. Analysis of intimal extent and predictors of renal atrophy in patients with aortic dissection. Acta Radiol 53(7):732-41.

Englberger L, Suri RM, Greason KL, Burkhart HM, Sundt TM 3rd, Daly RC, et al. 2011. Deep hypothermic circulatory arrest is not a risk factor for acute kidney injury in thoracic aortic surgery. J Thorac Cardiovasc Surg 141(2):552-8.

Garg AX, Devereaux PJ, Yusuf S, Cuerden MS, Parikh CR, Coca SG, et al. 2014. Kidney function after off-pump or on-pump coronary artery bypass graft surgery: a randomized clinical trial. JAMA 311(21):2191-8.

Haase M, Mertens PR. 2015. Biomarkers: more than just markers! Nephrol Dial Transplant 30(1):33-8.

Ho J, Lucy M, Krokhin O, Hayglass K, Pascoe E, Darroch G, et al. 2009. Mass spectrometry-based proteomic analysis of urine in acute kidney injury following cardiopulmonary bypass: a nested case-control study. Am J Kidney Dis 53(4):584-95.

Hoogmoed RC, Patel HJ, Kim KM, Williams DM, Deeb GM, Yang B. 2019. Acute Kidney Injury in Acute Type B Aortic Dissection: Outcomes Over 20 Years. Ann Thorac Surg 107(2):486-92.

Karkouti K. 2012. Transfusion and risk of acute kidney injury in cardiac surgery. Br J Anaesth 109 Suppl 1:i29-i38.

Khan UA, Coca SG, Hong K, Koyner JL, Garg AX, Passik CS, et al. 2014. Blood transfusions are associated with urinary biomarkers of kidney injury in cardiac surgery. J Thorac Cardiovasc Surg 148(2):726-32.

Kilic A, Whitman GJ. 2014. Blood transfusions in cardiac surgery: indications, risks, and conservation strategies. Ann Thorac Surg 97(2):726-34.

Kindzelski BA, Corcoran P, Siegenthaler MP, Horvath KA. 2018. Postoperative acute kidney injury following intraoperative blood product transfusions during cardiac surgery. Perfusion 33(1):62-70.

Klein SJ, Brandtner AK, Lehner GF, Ulmer H, Bagshaw SM, Wiedermann
CJ, et al. 2018. Biomarkers for prediction of renal replacement therapy in acute kidney injury: a systematic reviewand meta-analysis. Intensive Care Med 44(3):323-36.

Ko T, Higashitani M, Sato A, Uemura Y, Norimatsu T, Mahara K, et al. 2015. Impact of Acute Kidney Injury on Early to Long-Term Outcomes in Patients Who Underwent Surgery for Type A Acute Aortic Dissection. Am J Cardiol 116(3):463-8.

Kumar AB, Suneja M. 2011. Cardiopulmonary bypass-associated acute kidney injury. Anesthesiology 114(4):964-70.

Kumar AB, Suneja M, Bayman EO, Weide GD, Tarasi M. 2012. Association between postoperative acute kidney injury and duration of cardiopulmonary bypass: a meta-analysis. J Cardiothorac Vasc Anesth 26(1):64-9.

Ma WG, Zheng J, Zhang W, Sun K, Ziganshin BA, Wang LF, et al. 2014. Frozen elephant trunk with total arch replacement for type A aortic dissections: Does acuity affect operative mortality? J Thorac Cardiovasc Surg 148(3):963-70.

Park JT. 2017. Postoperative acute kidney injury. Korean J Anesthesiol 70(3):258-66.

Redahan L, Murray PT. 2017. Biomarkers of drug-induced kidney injury. Curr Opin Crit Care 23(6):463-9.

Rosner MH, Okusa MD. 2006. Acute kidney injury associated with cardiac surgery. Clin J Am Soc Nephrol 1(1):19-32.

Vercaemst L. 2008. Hemolysis in cardiac surgery patients undergoing cardiopulmonary bypass: a review in search of a treatment algorithm. J Extra Corpor Technol 40(4):257-67.

Wang CC, Lin HS, Huang YL, Wu FZ, Chuo CC, Ju YJ, et al. 2018. Renal artery involvement in acute aortic dissection: Prevalence and impact on renal atrophy in non-interventional treatment patients. J Cardiovasc Comput Tomogr 12(5):404-10.

Wasung ME, Chawla LS, Madero M. 2015. Biomarkers of renal function, which and when? Clin Chim Acta 438:350-57.

Wu HB, Qin H, Ma WG, Zhao HL, Zheng J, Li JR, et al. 2017. Can Renal Resistive Index Predict Acute Kidney Injury After Acute Type A Aortic Dissection Repair? Ann Thorac Surg 104(5):1583-9.

Zhou H, Wang G, Yang L, Shi S, Li J, Wang M, et al. 2018. Acute Kidney Injury after Total Arch Replacement Combined With Frozen Elephant Trunk Implantation: Incidence, Risk Factors, and Outcome. J Cardiothorac Vasc Anesth 32(5):2210-7.

Zindovic I, Gudbjartsson T, Ahlsson A, Fuglsang S, Gunn J, Hansson EC, et al. 2019. Malperfusion in acute type A aortic dissection: An update from the Nordic Consortium for Acute Type A Aortic Dissection. J Thorac Cardiovasc Surg 157(4):1324-33. 